\title{
Determinantes de la adopción de TIC en países en vía de desarrollo: el caso de las empresas industriales ecuatorianas ${ }^{1}$
}

\author{
Héctor Alberto Botello Peñaloza ${ }^{2}$ \& Aura Cecilia Pedraza Avella 3 \\ Universidad Industrial de Santander, Colombia
}

Recibido, abril 24 de 2015

Concepto evaluación, julio 6 de 2015

Aceptado, octubre 15 de 2015
Referencia: Botello Peñaloza, H.; Pedraza Avella, A. (2015). "Determinantes de la adopción de TIC en países en vías de desarrollo: el caso de las empresas industriales ecuatorianas". Revista Academia y Virtualidad, 8, (2), 48-59

\section{Resumen}

Este trabajo investiga los determinantes de la adopción de las tecnologías de la información y de la comunicación (TIC) en el sector industrial del Ecuador con base en datos de 14,324 empresas dentro del censo industrial de 2010. Con este fin, se utiliza un modelo Probit que estima la probabilidad de la implementación de TIC en función de una serie de características propias de las firmas. En general, la adopción de TIC en las empresas industriales en Ecuador es significativamente baja. Sólo $11 \%$ poseen una conexión a internet, 13\% cuentan con correo electrónico y apenas un $3 \%$ con página web.

Los volúmenes de ventas junto con el tamaño de la firma son los factores que más influyen en la difusión de estos componentes dentro de las empresas. Le siguen el grado de formalidad de la empresa, la localización de la firma en grandes ciudades y la pertenencia a sectores que elaboren bienes de capital.

Palabras clave: tecnologías de la información, difusión tecnológica, modelo Probit, empresas industriales, Ecuador.

\section{Key factors to adopt ICTs at developing countries: an experience of Ecuadorian industrial firms}

\begin{abstract}
This paper researches the key issues to adopt information and communication technologies (ICT) at the Ecuadorian industrial area based on 2010 census data of 14,324 industrial firms. To do that, we used a Probit model estimating ICT implementation

1. Artículo de investigación.

2. Profesor cátedra Política Monetaria y Fiscal, Universidad Industrial de Santander. Candidato a Maestría Ingeniería Industrial, Universidad Industrial de Santander. Correo: hectoralbertobotello@gmail.com

3. Profesora Universidad Industrial de Santander. Doctora Ciencias Económicas, Escuela de Estudios Industriales y Empresariales. Grupo Finance y Management, Bucaramanga. Correo: acecipe@uis.edu.co
\end{abstract}


Héctor Alberto Botello Peñaloza \& Aura Cecilia Pedraza Avella

probability based on a series of its own features. All together, the adoption of ICT of industrial enterprises in Ecuador is considerably low; only 11 percent has internet connection, email 13 percent, and 3 percent website.

Sales volumes along with size of firms are factors influencing the diffusion of these key issues within firms. The conventionalism, the location of the firm in large cities, and sectors developing capital goods are the other key factors to take into account.

Keywords: information technologies, technological diffusion, Probit model, industrial firms, Ecuador.

\section{Determinantes da adopção de TIC em países em via de desenvolvimento: o caso das empresas industriais equatorianas}

\section{Resumo}

Este trabalho pesquisa os determinantes da adopção das tecnologias da informação e da comunicação (TIC) no setor industrial do Equador com base nos dados de 14,324 empresas dentro do censo industrial de 2010. Com esse fim, utiliza-se o modelo Probit que estima a probabilidade da implementação de TIC em função de uma série de caraterísticas próprias das firmas. Em geral, a adopção de TIC nas empresas industriais no Equador é significativamente baixa. Só 11\% possuem uma conexão internet, $13 \%$ contam com correio eletrônico e apenas um 3\% com página web.

Os volumes de vendas junto com o tamanho da firma são os fatores que mais influem na difusão destes componentes dentro das empresas. Lhe seguem o grau de formalidade da empresa, a localização da firma em grandes cidades e a pertença a setores que elaborem bens de capital.

Palavras chave: tecnologias da informação, difusão tecnológica modelo Probit, empresas industriais, Equador.

\section{Introducción}

Actualmente los avances tecnológicos dentro de la sociedad del conocimiento afectan de manera significativa las relaciones que se comparten entre proveedores, distribuidores, competidores, clientes y los mismos procesos de manufactura. Esta nueva estructura es el principal desafío del siglo XXI para las empresas (David, 2003:93), lo que les da la oportunidad de producir nuevos productos o procesos en pro de ganar participación en el mercado.

El entendimiento principal para afrontar esta problemática es la idea con respecto a que la información y el conocimiento ahora constituyen insumos relevantes dentro de las empresas. En este sentido, las tecnologías de información y comunicación (TIC) surgen como la elección preferida para manipularla. Su efecto es una mejora en la comunicación y en la organización de datos dentro de la empresa. Esto contribuye a los aumentos de la productividad por la mayor creación de valor para clientes y de ventaja sobre la competencia (Slywotzky y Morrison, 2001).

En este sentido, es importante entender los procesos que facilitan la adopción de las TIC dentro de las empresas y el presente trabajo contribuye a los escasos aportes en Latinoamérica sobre este tema, explorando específicamente el sector industrial del Ecuador, manejando datos a nivel de firmas tomados del censo económico de 2010.

Para lo anterior, el artículo se organiza de la siguiente manera. En la primera, se exploran los fundamentos 
Determinantes de la adopción de TIC en países en vía de desarrollo: el caso de las empresas industriales ecuatorianas

teóricos de las TIC y los determinantes principales para su adopción dentro de la empresa, describiendo las principales contribuciones al tema hallados en la literatura internacional. En la sección 2 se explica la metodología y los datos por utilizar. Posteriormente, en la sección 3 se presentan los resultados de las estimaciones realizadas. Finalmente, en la sección 4 y 5 , respectivamente, se exponen las conclusiones y las referencias.

\section{Marco teórico}

Las definiciones de TIC son sumamente variadas, sin embargo es posible encontrar parámetros similares entre todas ellas. Por ejemplo, la Asociación Americana de las Tecnologías de la Información (ITAA) las expone como procesos a través de los cuales se administra, mantiene y diseña la información; ejemplos de lo anterior son los sistemas informáticos, teléfonos celulares, internet, periódicos digitales, etc. Rebolloso (2000: 66) usa el término TIC para referirse a los medios colectivos para reunir y luego almacenar, transferir, procesar y recuperar electrónicamente información, tales como palabras, números, imágenes y sonidos. Entre tanto, Barragán (2002: 188) y González et al. (1996: 413) coinciden en que las TIC abarcan cualquier tipo de hardware o software operados para captura, procesamiento y presentación de datos utilizados por cualquier empresa que manipula información.

En la empresa, la aplicación de las TIC se muestra en tres áreas principales: la comunicación, la clasificación y la transición de la información. Una información bien canalizada y precisa puede incrementar la eficiencia en los procesos empresariales disminuyendo los costos de producción (Alderete y Gutiérrez, 2012). En este sentido, la difusión de las TIC causa mejoras sustanciales a través de todos los canales dentro de la organización. Gi-Soon (2005) y Huaroto (2012) describen (ver figura 1) los primeros impactos en los tiempo de procesamiento y comunicación de la información, que derivan en menores costos de transacción y mejores decisiones de los involucrados tanto empleados como gerentes. Al final, estas mejoras las reciben los clientes en forma de un mejor servicio incrementando las ventas, generando

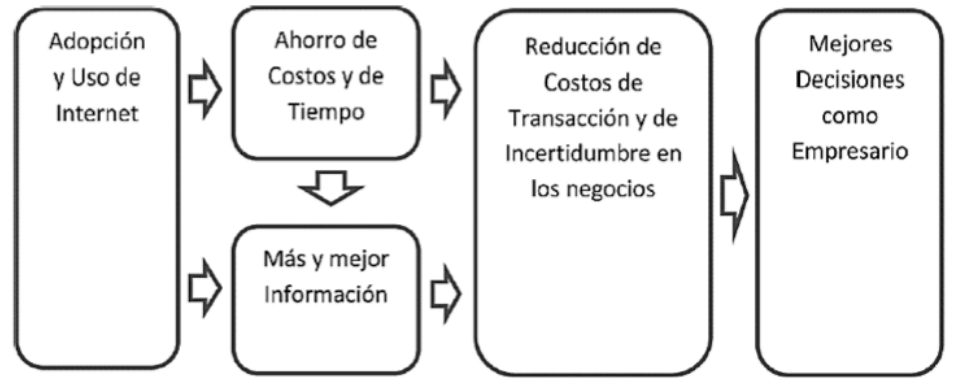

Figura 1. Relación causal: efecto del uso de internet en la toma de decisiones Fuente: Gi-Soon (2005); elaboración de Huaroto (2012)

un crecimiento de la producción y retroalimentando un ecosistema virtuoso (Sellens, 2006).

Ahora bien, entre los factores que favorecen la difusión de las TIC dentro de las organizaciones están:

- Los conocimientos que posean los integrantes de la organización: mayor dotación de saberes técnicos sobre las aplicaciones por integrar dentro de los procesos organizativos, permite una difusión más rápida y fácil de las tecnologías (Chun, 2003; Hollestein, 2004).

- El tamaño: la literatura sugiere que debido a los diferentes recursos humanos y tecnológicos que deben costear las empresas para la adopción de TIC, muchas no se pueden permitir su implementación en especial las pequeñas donde los recursos para la inversión son relativamente escasos (Geroski, 2000; Alderete, Jones y Morero, 2013). Al mismo tiempo, es más probable que los directores de las firmas más pequeñas no puedan valorar correctamente los beneficios que les puede propiciar el uso de las TIC en el largo plazo (ALADI, 2005). Sin embargo, otros grupos de autores afirman que en un contexto de globalización, el tamaño ya no es un elemento clave para su implementación debido a la reducción continua de los costos (Vilaseca et al, 2002).

- La edad: la experiencia y antigüedad que tenga la firma en los mercados puede implicar, según Hollenstein (2004), un arma de doble filo. Por un lado, las que poseen un mayor tiempo de recorrido pueden entender mejor cómo las TIC se pueden integrar en la orga- 
Héctor Alberto Botello Peñaloza \& Aura Cecilia Pedraza Avella

nización, también podría impedir su adopción dado la resistencia al cambio de los directores.

- La internacionalización de la empresa y la pertenencia de colaboraciones externas puede incrementar la probabilidad de incursionar en el comercio electrónico (Daniel y Grimshaw, 2002; Wagner et al, 2003).

En cambio, el costo de la tecnología, la falta de habilidades gerenciales y de recursos financieros pueden ser factores que inhiban la implementación de TIC (McCole y Ramsey, 2005). Igualmente, si no hay una idea estructurada dentro de la organización de cómo las TIC se combinarán con los procesos actuales, la puesta en marcha de estos recursos no tendrá ningún efecto en la competitividad (Peirano y Suárez, 2004).

En los trabajos aplicados que exploran la influencia de estos determinantes a nivel de las empresas de cada uno de los países, se observa que Europa es la región con la mayor cantidad de estudios. Entre los más relevantes se tienen el de Fabiani, Schivardi y Trento (2005). Estos autores exploran la adopción de TIC en 1.500 empresas manufactureras italianas, investigando si la presencia de algunas características internas y externas favorecen o ralentizan este fenómeno.

La variable de medición a través de un modelo de regresión lineal fueron los gastos en inversiones en TIC. Encuentran que los factores más importantes son el tamaño, el capital humano calificado y la presencia de grandes conglomerados empresariales en el medio ambiente local.

Galliano, Roux y Filippi, (2001) manejan un conjunto de datos de aproximadamente 5.000 empresas industriales francesas. A través de un modelo de medias combinan variables internos y externos de las empresas; como por ejemplo la idea de la integración que tienen los gerentes y las relaciones que poseen con sus entornos espaciales (áreas urbanas o rurales). Más allá de los determinantes tradicionales, los autores confirman que los factores organizacionales desempeñan un papel importante en la adopción de las TIC. Asimismo, resaltan la importancia de la interacción de la organización con el tipo de zonas donde se encuentra, enfatizando la necesidad de apartarse de una visión de la empresa como una entidad de una sola unidad.

Por su parte, la investigación de Bayo y Lera (2007) analizó en 337 centros de trabajo de España el efecto que tenía el medio externo, las características estructurales de las firmas, capital humano, estrategia competitiva y la disposición interna en la adopción de las TIC. Entre los resultados se observa que las variables que impactan de mayor manera son el tamaño del establecimiento, la propiedad multinacional y la mano de obra calificada.

En Irlanda, Haller y Siedschlag (2011) estudiaron entre 2001 y 2004 a empresas manufactureras y su adopción de TIC. Los resultados sugieren que si las firmas que son más grandes, exportadoras, jóvenes, y han experimentado un rápido crecimiento en sus ventas, tienen mayor tendencia a la utilización de TIC. Sin embargo, es necesaria la presencia de ciertos niveles de cualificación de la mano de obra para desarrollar spillovers tecnológicos positivos dentro y fueron de las empresas.

Con una muestra de 115 empresas de logística de la Asociación de Naciones del Sudeste Asiático (ASEAN), Tongzon y Hong-Oanh (2013) estimaron un modelo Logit para evaluar la adopción de las TIC en función de un conjunto de características de las empresas. Encuentran que entre países, los niveles de adopción en TIC pueden ser significativas dadas las diferencias en la composición sectorial de los países.

En Latinoamérica, la evidencia empírica a nivel de empresa en relación con los países europeos es bastante limitada y reciente. En Colombia, Gallego, Gutiérrez y Lee (2011) investigaron, con datos de 2006, la difusión de TIC dentro de 3,759 empresas manufactureras. Manejando modelos de regresión lineal, los autores encuentran que la implementación de TIC se facilita cuando el establecimiento es relativamente grande, tiene un gran capital humano, se dedica a actividades más innovadoras y cuando la estructura organizativa interna está más alineada con una tecnología dada. 
Alderete, Jones, Morero (2013) investigan datos de pequeñas y medianas empresas $(83 \%)$ de la industria automotriz y siderúrgica de Argentina. Los resultados muestran que los factores que influyen positivamente en la adopción de las TIC son la pertenencia a un gremio económico, el nivel global de innovación y el nivel de cualificación de los empleados.

\section{Metodología}

\section{Fuentes de datos}

El censo económico de Ecuador realizado en 2010 es la fuente principal de los datos de este artículo. Consiste en un conteo de todas las unidades económicas que conforman el sector productivo, su ubicación, así como el registro de sus características principales. Se lleva a cabo mediante una serie de visitas a los establecimientos económicos.

La información se pide a la persona propietaria o administradora del establecimiento. Las visitas se realizaron de septiembre a noviembre de 2010. En total se encuestaron cerca de 47,867 empresas industriales que emplean a 267 mil personas, distribuidas por los siguientes sectores económicos.

\begin{tabular}{|l|c|c|c|c|}
\hline Sectores industriales & $\begin{array}{c}\mathrm{N}^{\circ} \text { de } \\
\text { empresas }\end{array}$ & $\%$ & Personal ocupado & $\%$ \\
\hline Total & 47,867 & $100 \%$ & 266,908 & $100 \%$ \\
Productos alimenticios & 9838 & $21 \%$ & 72,865 & $27 \%$ \\
Prendas de vestir & 8273 & $17 \%$ & 28,202 & $11 \%$ \\
Productos elaborados de metal & 8021 & $17 \%$ & 21,572 & $8 \%$ \\
Muebles & 5663 & $12 \%$ & 19,023 & $7 \%$ \\
Madera y madera y corcho & 3109 & $6 \%$ & 10,857 & $4 \%$ \\
Otros productos minerales no & 2614 & $5 \%$ & 15,664 & $6 \%$ \\
metálicos & 2001 & $4 \%$ & 11,034 & $4 \%$ \\
Impresión y regrabaciones & 1466 & $3 \%$ & 10,110 & $4 \%$ \\
Productos textiles & 1355 & $3 \%$ & 5,950 & $2 \%$ \\
Reparación de maquinaria y equipo & 1254 & $3 \%$ & 8,077 & $3 \%$ \\
Cueros y productos conexos & 507 & $1 \%$ & 12,119 & $5 \%$ \\
Caucho y plástico & 404 & $1 \%$ & 3,163 & $1 \%$ \\
Maquinaria y equipo n.c.p. & 401 & $1 \%$ & 5,194 & $2 \%$ \\
Vehículos automotores, y remolques & 365 & $1 \%$ & 6,323 & $2 \%$ \\
Substancias y productos químicos & 268 & $1 \%$ & 6,613 & $2 \%$ \\
Papel y de papel & 224 & $0 \%$ & 2,734 & $1 \%$ \\
Metales comunes & 202 & $0 \%$ & 8,458 & $3 \%$ \\
Bebidas & 160 & $0 \%$ & 4,009 & $2 \%$ \\
Equipo eléctrico & 1742 & $4 \%$ & 14,941 & $6 \%$ \\
Otras & & & & \\
\hline
\end{tabular}

Tabla 1. Sectores industriales censados en el Ecuador en 2010

Fuente: cálculos del autor con base en los datos del censo económico de Ecuador, 2010, llevado a cabo por el Instituto de Estadística del Ecuador (INEC)
Las empresas de productos alimenticios constituyeron $27 \%$ de los establecimientos encuestados y el mismo porcentaje de personas ocupadas, seguidas por las firmas que fabrican prendas de vestir con $17 \%$ y $11 \%$, respectivamente.

\section{Modelo de elección discreta}

En la literatura inicial sobre difusión de TIC en las empresas se ha utilizado frecuentemente el análisis de regresión múltiple, el cual consiste en intentar determinar el comportamiento de una variable continua en función de una o varias variables independientes. Algunos ejemplos son Hargittai (1999) (conexión a internet); Kiiski y Pohjola (2002) (servidores de internet); Chinn y Fairlie (2007) (los usuarios de computadoras personalesPC e internet). Sin embargo, algunos autores, tales como Wolcott et al. (2001) y Billon, Marco y Lera-López (2009), han señalado que el análisis de una sola tecnología por separado no aporta mucha información sobre el nivel de desarrollo digital dentro de una empresa.

Por esto, este trabajo busca aportar a la literatura relacionada recurriendo a los modelos de elección discreta. Éstos se aplican a variables cuyos valores son dicotómicos y buscan estimar la probabilidad que suceda cierto fenómeno en función de unas características determinadas y tal como sea la función observada existen los modelos de probabilidad lineales truncados, el modelo Logit y el modelo Probit.

En general se considera que detrás de la variable dependiente en estos modelos, $\mathrm{Y}$, se encuentra una variable no observable, I, que depende de un conjunto de variables explicativas, $\mathrm{Xi}$, que toma ciertos valores si ha sobrepasado cierto lumbral, tal como se expresa a continuación.

$$
Y_{i}=\left\{\begin{array}{lll}
1 & \text { si } I_{i}^{*}>0 & \text { lo que ocurre cuando } X_{i} \beta+\varepsilon_{i}>0 \\
0 & \text { si } I_{i}^{*}<0 & \text { lo que ocurre cuando } X_{i} \beta+\varepsilon_{i}<0
\end{array}\right.
$$


Héctor Alberto Botello Peñaloza \& Aura Cecilia Pedraza Avella

Donde el supuesto sobre la distribución de $\varepsilon$ determina el tipo de modelo a estimar. Si se supone una función de distribución uniforme, se utiliza el modelo lineal de probabilidad truncado; si se distribuye como una normal con media cero y varianza uno, el modelo generado será un Probit; mientras que si se supone que se distribuye como una curva logística, se trataría de un modelo Logit. La hipótesis de que el umbral a superar por la variable latente sea cero, se puede modificar por cualquier otro valor sugiriéndose, en determinados estudios, que el valor crítico sea el definido por el término constante (Pérez, 2004).

Bajo el primer enfoque el modelo probabilístico quedaría definido (Medina, 2003)

$$
P_{i}=\operatorname{Pr} o b\left(Y_{i}=1\right)=\operatorname{Pr} o b\left(I_{i}^{*}>0\right)=\operatorname{Pr} o b\left(X_{i} \beta+\varepsilon_{i}>0\right)=F\left(X_{i} \beta\right)
$$

Con el modelo así definido ${ }^{4}$, la variable endógena del modelo dicotómico representa la probabilidad de ocurrencia del fenómeno analizado. Para este trabajo, la función de estimación probabilística será

$$
\mathrm{Y}=\mathrm{f}(\mathrm{S}, \mathrm{X}) \quad \mathrm{Y}=\alpha+\beta \mathrm{Xi}+\varepsilon
$$

Donde Y es una variable dicotómica, 1 corresponde a la implementación del componente TIC y cero en el caso contrario. Ésta es explicada por un vector de variables $\mathrm{X}$ de la empresa. En su interpretación, $\beta$ captaría el efecto marginal que aporta cada uno de estos factores a la probabilidad de implementar las tecnologías.

La efectividad de estos modelos se mide a través de la clasificación correcta de los individuos dada sus probabilidades de participar o no en el fenómeno analizado, que se logra con ayuda de las variables independientes. Este indicador se denomina pseudo $\mathrm{R}^{2}$. Cuanto más alto, más efectivo es el modelo en determinar el comportamiento de la variable dependiente.

\section{Implementación de TIC}

Estudiar la implementación de TIC en las empresas es un fenómeno complejo (Verdegem y Lieven, 2011) debido a la multitud de formas que pueden adoptar. En este trabajo se evalúa el impacto que pueda tener la implementación de las siguientes tecnologías:

- La conexión a internet

- Tenencia de página web

- Cuenta de correo electrónico oficial desde donde contacta a clientes y proveedores

- Tenencia de una máquina fax

- Tenencia de línea de número de teléfono oficial desde donde contacta a clientes y proveedores.

Éstos se recopilan en los datos en forma de variables dicotómicas. En este sentido, para poder incluirlas en el modelo de elección discreta, se crea un indicador normalizado continuo en línea con la metodología de Lefebvre y Lefebvre (1996), permitiendo una mejor comprensión del potencial de las TIC dentro de los establecimientos productivos (Huaroto, 2012). Este indicador toma los valores de cero a cien, denominándose índice TIC. A continuación se organiza como una variable dicotómica de la siguiente manera.

- 0 si índice TIC $<0.50$

- 1 si $0.50<$ índice TIC $<1$

Entre tanto, las variables independientes serán características de las firmas, tales como:

- Años de operación de la empresa

- Consumo de $\mathrm{kW} / \mathrm{hora}$

- Sexo del gerente

4. Para un desarrollo más completo de los modelos relacionados, léase Pérez (2004). 
Determinantes de la adopción de TIC en países en vía de desarrollo: el caso de las empresas industriales ecuatorianas

- Naturaleza jurídica del establecimiento

- Afiliación a un gremio empresarial

- Capacitación de los empleados

- Grado de formalidad de la firma (tenencia del RUC, registros contables)

- Número de empleados (hombres, mujeres, remunerados, temporales etc.)

- Provincia

A continuación se expone un breve análisis estadístico descriptivo de la implementación de TIC en el sector industrial ecuatoriano.

\section{Resultados y análisis}

\section{Estadísticas descriptivas alrededor de las TIC}

La tenencia de internet por provincias se observa en el figura 2. En total 10,89\% de las firmas tienen una conexión a internet. Las empresas con más de 50 empleados son las de mayor penetración, por encima de $88 \%$. Sin embargo, es de resaltar que $94 \%$ de los establecimientos en el Ecuador tienen menos de 10 empleados y en su tenencia de internet es sólo de 7,71. Por provincias, la penetración del internet en Pichincha, Guayas, Azuay y Orellana se encuentra por encima de $11,36 \%$. La causa de lo anterior es que en estas provincias se ubican las ciudades más grandes del Ecuador, tales como Quito y Guayaquil, lo que se encuentra favorecido la concentración de los proveedores de infraestructura TIC y, por ende, la mayor adaptación por parte de las empresas.

En relación con otros componentes TIC, se aprecia una baja tenencia del fax, el correo electrónico y de las páginas web dentro la empresa, con 9,27\%, 13,18\% y 2,77\%. Además se observa una correlación directa consistente con tamaño de la firma: con más empleados, mayor es la penetración de TIC. Por ejemplo, todas las empresas de más de 500 empleados poseen una máquina fax, frente a solo $6 \%$ de las empresas con menos de 10 empleados.
Tenencia de Internet por Tamaño de la firma

\begin{tabular}{|c|c|}
\hline Personal & $\begin{array}{c}\text { \% de empresas } \\
\text { con internet }\end{array}$ \\
\hline 1 a 9 & 7.71 \\
\hline $10 \mathrm{a} 49$ & 68.84 \\
\hline $50-99$ & 90.22 \\
\hline $100-199$ & 88.64 \\
\hline $200-499$ & 88.00 \\
\hline$>500$ & 96.97 \\
\hline Total & 10.89 \\
\hline
\end{tabular}

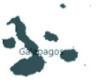

Figura 2. Porcentaje de empresas industriales con conexión a internet, Ecuador, 2010

Fuente: Cálculos del Autor con base en los datos del censo económico de Ecuador 2010 llevado a cabo por el Instituto de Estadística del Ecuador (INEC)

\begin{tabular}{|l|l|l|l|l|}
\hline Personal & $\begin{array}{l}\text { Correo } \\
\text { electrónico }\end{array}$ & Web & Fax & $\begin{array}{l}\text { Teléfono } \\
\text { fijo }\end{array}$ \\
\hline 1 a 9 & 10.15 & 1.13 & 5.93 & 82.93 \\
\hline 10 a 49 & 64.51 & 23.2 & 62.81 & 97.48 \\
\hline 50 - 99 & 80.22 & 45.9 & 82.84 & 99.25 \\
\hline $100-199$ & 72.67 & 63.35 & 82.61 & 99.38 \\
\hline $200-499$ & 66.67 & 68.18 & 84.09 & 100 \\
\hline$>500$ & 57.14 & 71.43 & 100 & 100 \\
\hline Total & 13.18 & 2.77 & 9.27 & 83.74 \\
\hline
\end{tabular}

Tabla 2. Tenencia de otros componentes constitutivos de las TIC por tamaño de la empresa. Porcentaje de empresas industriales.

Fuente: cálculos del autor con base en datos del Censo Económico de Ecuador, 2010, llevado a cabo por el Instituto de Estadística del Ecuador (INEC)

\section{Adopción de TIC: modelo probabilístico}

Después de depurar la base de datos y extraer las empresas que no respondieron correctamente al censo, se analizaron las características de cerca de 14,324 firmas. En la tabla 3 se exponen los resultados de las estimaciones de la ecuación 1 con las variables seleccionadas mediante los datos disponibles para las empresas dentro del censo económico realizado por el INEC en 2010. Se muestran las observaciones totales, el pseudo $\mathrm{R}^{2}$ y las variables categóricas, las características base evaluadas, los coeficientes originales de la distribución y el efecto marginal porcentual que tienen sobre las variables dependientes con su nivel de significancia. 
Héctor Alberto Botello Peñaloza \& Aura Cecilia Pedraza Avella

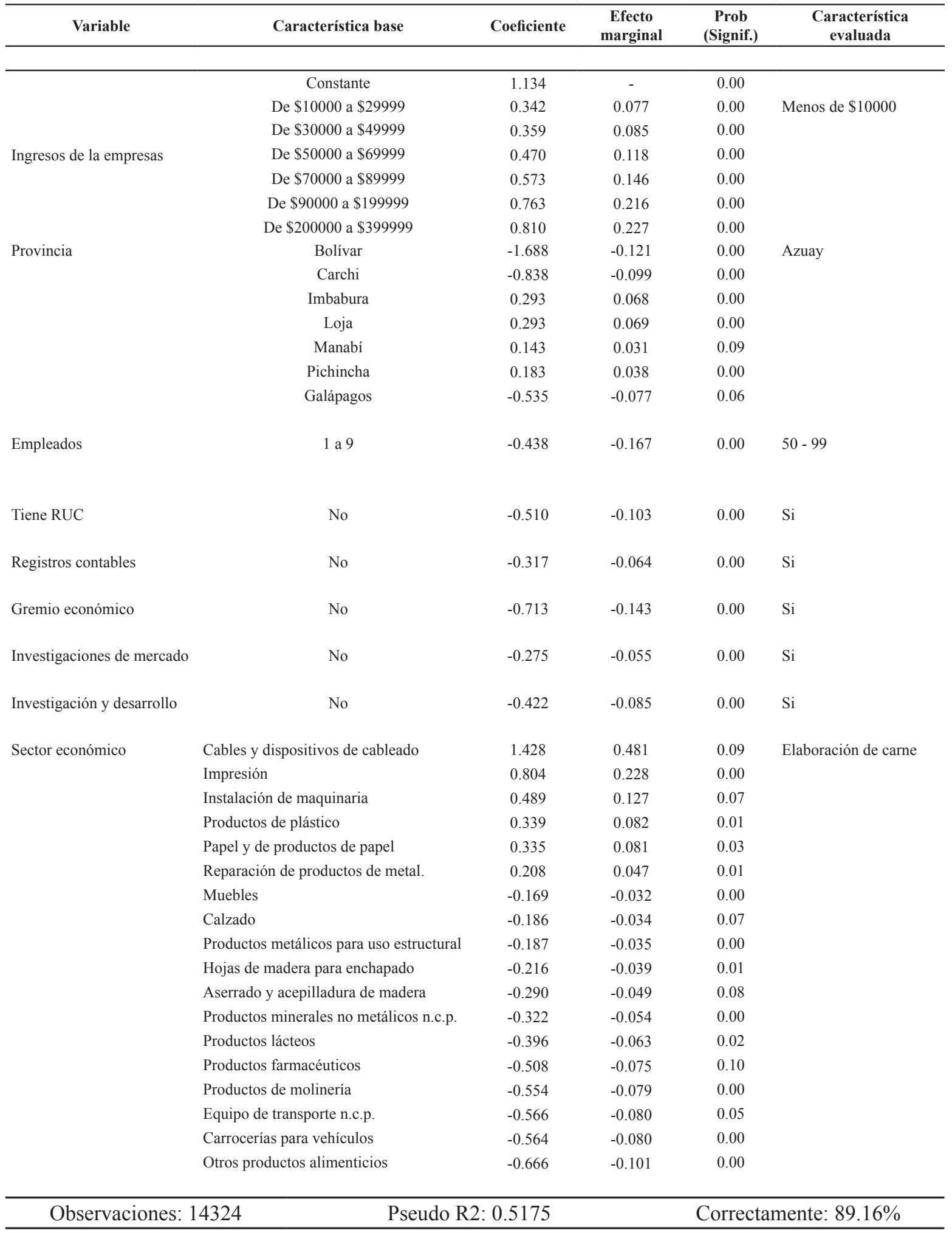

Fuente: cálculos del autor con base en datos del Censo Económico de Ecuador, 2010, llevado a cabo por el Instituto de Estadística del Ecuador (INEC) 
Determinantes de la adopción de TIC en países en vía de desarrollo: el caso de las empresas industriales ecuatorianas

Se observa que todas las variables introducidas en el modelo son significativas, $5 \%$. El porcentaje de varianza estimado para el modelo de las ventas es cercano a $51,75 \%$, mientras que el porcentaje de calificación correcta por parte del modelo alcanzó 89\% de las observaciones. Esto se debe principalmente a la introducción de suficientes variables explicativas y al comportamiento en la varianza de cada uno de los datos.

Se aprecia que los niveles de ventas es la variable que influencia en mayor medida la tenencia o no de TIC. En este sentido, si la empresa tiene ventas entre US\$10 mil y 30 mil, existe una probabilidad adicional de 7,7\% en adoptar TIC en contraposición de las que facturan menos de 10 mil. Así sucesivamente, hasta el estrato más alto de ventas que está entre US\$200 mil y 400 mil, con una probabilidad marginal adicional de $22,7 \%$. Las estimaciones también muestran que las pequeñas empresas del Ecuador (menos de 10 empleados) tienen hasta $16,7 \%$ menos de probabilidad de adoptar TIC que las empresas de 50 a 99 personas. Esto puede ser, tal y como lo menciona Geroski (2000), debido a los altos costos de la implementación de TIC además de la capacitación necesaria para la puesta en marcha de las mismas.

A continuación se aprecia que en algunas provincias de Ecuador, la probabilidad de adoptar TIC es más alta que en otras. En este sentido, Loja (6,9\%), Imbabura $(6,8 \%)$ y Pichincha $(3,8 \%)$ son las que enseñan los lugares donde las firmas industriales pueden adoptar las TIC con mayor facilidad dado que son provincias con ciudades relativamente grandes donde se concentran las prestadoras de servicios de telecomunicaciones. Parece que la afirmación de Galliano, Roux y Filippi (2001), sobre las interacciones de la organización con su medio externo, también tiene una importancia significativa en los países en vías de desarrollo.

Es interesante notar que la antigüedad de la empresa no fue una de las variables significativa dentro del modelo. Esto puede confirmar que en el mercado actual, la experiencia de la firma dentro de un mercado no es una barrera que limite la difusión de TIC dentro de las mismas.
La informalidad empresarial también impacta negativamente la adopción de TIC dentro de las empresas. En este sentido, si las empresas no tienen registros contables, ni registro de actividad (RUC) la probabilidad de que adopten TIC se reduce en un 16,7\%. Mientras tanto la afiliación a un gremio económico afecta positivamente un $14,3 \%$ la posibilidad de que la empresa implemente TIC. Según Fabiani, Schivardi y Trento (2005) la pertenencia a estos grupos favorece a las empresas en adquirir capacitación y estar en el foco de atención por parte de los principales proveedores de telecomunicaciones para poder expandir su actividad productiva.

En línea con lo anterior, las empresas que poseen una actividad innovadora activa, tales como departamentos de mercadeo y de innovación, aumentan la probabilidad de implementar TIC en su establecimiento. Según De La Fuente Sabaté y Del Canto (2003), estas firmas desarrollan la capacidad de conocer mejor las necesidades de sus clientes y el papel que tiene la empresa en el mercado. En este sentido, estas actividades son más efectivas si están sustentadas en una aplicación intensiva de las TIC. Cuantitativamente, el aporte marginal de ambas características es $14 \%$.

Igualmente desde el punto de vista sectorial y dadas las características de una economía dual como la presente en el Ecuador (López y Niklitschek, 1991), hay algunos sectores intensivos en capital que poseen una mayor tendencia a adoptar TIC que otros. Por ejemplo, sectores que enseñan las más altas probabilidades positivas son fabricación de cables, impresión, instalación de maquinaria con $48 \%, 22 \%$ y $12 \%$, respectivamente.

Finalmente, al comparar los anteriores resultados se aprecia que la adopción de TIC en las empresas industriales del Ecuador está en línea con las encontradas en los países desarrollados, la diferencia radica en la penetración regional que tienen. Es de esperar que esta tendencia se amplíe en la medida en que se impulsa un aumento de la infraestructura tecnológica. 
Héctor Alberto Botello Peñaloza \& Aura Cecilia Pedraza Avella

\section{Conclusiones}

Las necesidades que demanda actualmente la sociedad del conocimiento a las empresas hacen que éstas respondan al nuevo contexto utilizando las herramientas disponibles. Este fenómeno impulsa la búsqueda de mayores niveles de competitividad mediante el uso de las tecnologías de información y comunicación (TIC) dado que permiten una captación, manejo y clasificación de la información disponible para la empresa.

En este sentido, el presente trabajo estimó los determinantes de la adopción de TIC en las empresas industriales de Ecuador en 2010 tomando información a nivel del censo económico elaborado por el INEC. El modelo Probit que se utilizó calculaba la probabilidad de adopción de TIC en función de algunas características de las firmas.

Entre los resultados se muestra una baja presencia de las TIC en las empresas industriales en el Ecuador, particularmente en las de menos de diez empleados, las cuales constituyen cerca de $95 \%$ del tejido empresarial en el Ecuador. Por componentes, solo $11 \%$ de las empresas tienen internet, $13 \%$ cuenta de correo electrónico, fax $9 \%$, y 3\% página web. En las estimaciones, se muestra que el volumen de ventas junto con el tamaño de la firma son los factores que más influyen para que la empresa implemente las TIC.

Le siguen el grado de formalidad de la empresa, la localización de la firma en grandes ciudades y la pertenencia a sectores que elaboren bienes de capital.

Estos resultados deberían hacer que las políticas públicas destinadas al apoyo de las empresas se orientasen a fomentar el uso de las TIC en las más pequeñas a través de procesos formalización, para que éstas puedan explotar su uso en los procesos de producción para así mejorar su competitividad.

Entre las limitaciones del trabajo se encuentran el no poder estudiar los conocimientos de los empleados en TIC como es lo sugerido por Bayo y Lera (2007). Tampoco las prospectivas e intenciones que tienen los directivos sobre la difusión de TIC dentro de los establecimientos, ya que es un aspecto ampliamente estudiado en la literatura. Finalmente, al igual que en Alderete, Jones, y Morero (2013), este trabajo sólo se enfocó en el sector industrial, por lo que las dinámicas en otros sectores de la economía pueden estar dirigidas por otros factores.

\section{Referencias}

ALADI (2005). Uso actual y potencial de las tecnologías de la información y las comunicaciones en el sector empresarial de los países miembros de la ALADI. ALADI/SEC/Estudio 170.

Alderete, M. V., Jones, C., y Morero, H. (2013)

Determinantes de la adopción de las tic en las tramas productivas automotriz y siderúrgica de argentina. 10th International Conference on Information Systems y Technology Management - CONTECSI Junio, 12 to 14, - São Paulo, Brazil.

Barragán, J., Pagán, J., y Brown, C. (2002). Administración de las pequeñas y medianas empresas, retos y problemas ante la nueva economía global. México: Trillas.

Bayo-Moriones, A. y Lera-López F (2007). A firm-level analysis of determinants of ICT adoption in Spain. Technovation 27, no. 6: 352-366.

Billon, M., Marco, R., y Lera-López, F. (2009). Disparities in ICT adoption: A multidimensional approach to study the cross-country digital divide. Telecommunications Policy, 33(10), 596-610.

Chinn, M. D., y Fairlie, R. W. (2007). The determinants of the Global Digital Divide: A cross-country analysis of computer y internet penetration. Oxford Economic Papers, 59, 16-44.

Chun, H. (2003). Information Technology y the Demand for Educated Workers: Disentangling the Impacts of Adoption versus Use. The Review of Economics y Statistics, 85(1), 1-8.

Daniel, E. y Grimshaw, D. (2002). An exploratory comparison of electronic commerce adoption in large y small 
Determinantes de la adopción de TIC en países en vía de desarrollo: el caso de las empresas industriales ecuatorianas

enterprises. Journal of Information Technology, 17(3), $133-147$.

David, F. (2003): Conceptos de administración estratégica. México: Pearson Prentice Hall.

De La Fuente Sabaté, J. M., y del Canto, J. G. (2003). El proceso de innovación de la empresa española: Identificación de patrones de innovación. Cuadernos de Economía y Dirección de la Empresa, (16), 145171.

Fabiani, Silvia, Fabiano Schivardi, y Sandro Trento (2005). ICT adoption in Italian manufacturing: firm-level evidence. Industrial y Corporate Change 14, no. 2: 225-249.

Gallego, J. M., Gutiérrez, L. H., y Lee, S. H. (2011). A firm-level analysis of ICT adoption in an emerging economy: Evidence from the Colombian manufacturing industries (No. 009155).

Galliano, D., Roux, P., y Filippi, M. (2001). Organizational y spatial determinants of ICT adoption: the case of French industrial firms. Environment y Planning A, 33(9), 1643-1664.

Geroski, P. A. (2000). Models of technology diffusion. Research policy, 29(4), 603-625.

Gi-Soon, S. (2005). The impact of information y communication technologies (ICTs) on rural households: A holistic approach applied to the case of Lao People's Democratic Republic. Jakarta: UNV/ UNDP.

González, A.; Gisbert, M.; Guillem, A.; Jiménez, B.; Lladó, F. y Rallo, R. (1996). Las nuevas tecnologías en la educación. En Salinas, Jesús et al. (eds.). Redes de comunicación, redes de aprendizaje. Universitat de les Illes Balears: Edutec'95, págs. 409•422.

Haller, Stefanie A., y Iulia Siedschlag (2011). Determinants of ICT adoption: Evidence from firm-level data. Applied Economics 43, no. 26: 3775-3788.

Hargittai, E. (1999). Weaving the Western Web: Explaining differences in Internet connectivity among OECD countries. Telecommunications Policy, 23, 701-718.

Hollenstein, H. (2004). Determinants of the adoption of Information y Communication Technologies (ICT). An empirical analysis based on firm level data for the Swiss business sector. Structural change and Economic dynamics, 15(3), 315-342.

Huaroto, C. (2012). El Uso de Internet y la Productividad de las Microempresas: Evidencias del Caso Peruano (2007-2010) (Español). Information Technologies y International Development, 8(4), pp-129.

Kiiski, S., y Pohjola, M. (2002). Cross-country diffusion of the Internet. Information Economics y Policy, 14, 297-310.

Lefebvre, E. y Lefebvre, L. (1996). Information y telecommunication technologies. The impact of their adoption on small y medium-sized enterprises. Ottawa: IDRC.

Lopez, R., y Niklitschek, M. (1991). Dual economic growth in poor tropical areas. Journal of Development Economics, 36(2), 189-211.

McCole, P. y Ramsey, E. (2005). A profile of adopters y nonadopters of e-Commerce in SME professional service firms. Australian Marketing Journal, 13 (1), 36-48.

Medina, E. (2003). Modelos de elección discreta. Publicaciones Económicas de la Universidad Autónoma de Madrid. España, 26.

Peirano, F. y Suarez, D. (2004). Estrategias empresariales de uso y aprovechamiento de las TICs por parte de las Pymes de Argentina en 2004. Artículo presentado en el 33 Simposio sobre la Sociedad de la Información, Córdoba, Argentina, setiembre 2004.

Pérez López, C. (2004). Técnicas de análisis multivariante de datos. Aplicaciones con SPSS, Madrid, Universidad Complutense de Madrid.

Rebolloso, R. (2000): La globalización y las nuevas tecnologías de información, México, D.F.: Trillas. 
Sellens J. (2006) TIC, productividad y crecimiento económico la contribución empírica de Jorgenson, Ho y Stiroh, de Joan Torrent. UOC Artículos: Revista sobre la sociedad del conocimiento, ISSN 1885-1541, Nº. 2.

Slywotzky, A., y Morrison, D. (2001). Becoming a digital business: it is not about technology. Strategy y Leadership, 29(2), 4-9.

Tan, K. S., Chong, S. C., Lin, B., y Eze, U. C. (2009). Internet-based ICT adoption: evidence from Malaysian SMEs. Industrial Management y Data Systems, 109(2), 224-244.

Tongzon, Jose, y Hong-Oanh Nguyen (2013). ICT Adoption among Logistics Companies in ASEAN Countries. Transport Reviews 33, no. 5: 548-569

Verdegem, Pieter, y Lieven De Marez (2011). Rethinking determinants of ICT acceptance: Towards an integrated y comprehensive overview. Technovation 31, no. 8 411-423.

Vilaseca, J.; Torrent, J. y Díaz, A. (2002). ICTs y strategic y organizational changes in Catalan business. A review of select evidence for Catalonia, Spain y international data. Working Paper Series WP02-001.

Wagner, B; Fillis, I. y Johansson, U. (2003). E-business y e-supply strategy in small y medium sized enterprises (SMEs). Supply Chain Management, 8(4), 343-354.

Wolcott, P., Press, L., Mchenry, W., Goodman, S., y Foster, W. (2001). A framework for assessing the global diffusion of the Internet. Journal of the Association for Information Systems, 2, 1-50. 